\title{
CRITERIA FOR UNIQUE METRIC LINES IN BANACH SPACES
}

\author{
E. Z. ANDALAFTE AND J. E. VALENTINE
}

\begin{abstract}
A metric space $M$ has the monotone property if for each point $p$ and line $L$ of $M$ the distance $p x$ between $p$ and a point $x$ of $L$ is monotone increasing as $x$ recedes along either half-line of $L$ determined by the foot of $p$ on $L$. It is shown that a Banach space (over the reals) has the monotone property if and only if it has unique metric lines. Using previously known results, additional equivalents of the monotone property are obtained and new proofs of some older criteria for unique metric lines result.
\end{abstract}

1. Introduction. A metric space $M$ is said to have the monotone property if for each point $p$ and metric line $L$ of $M$ the distance $p x$ between $p$ and a point $x$ of $L$ is monotone increasing as $x$ recedes along either halfline of $L$ determined by the foot of $p$ on $L$. (For definitions and notation see [2].) It is known that euclidean and hyperbolic spaces have the monotone property. Moreover, in [1] and [4] the monotone property has played an important role in the characterization of euclidean space. In a conversation with one of the authors, L. M. Blumenthal asked what one could say about Banach spaces (over the reals) with the monotone property. In this paper we prove that a Banach space (over the reals) has the monotone property if and only if it has unique metric lines. This provides a complete answer to the question of Blumenthal. Immediate consequences of this result are the equivalence of the monotone property and rotundity of the space, as well as a simple new proof of the well-known fact that a Banach space has unique metric lines if and only if its unit spheres are strictly convex.

2. Preliminaries. A useful characterization of spaces having the monotone property is provided by the following lemma.

LeMma 2.1. Let $M$ be a metric space containing a metric line. The space $M$ has the monotone property if and only if $M$ does not contain a quadruple of points $p, q, r, s$ such that $q, r, s$ are on a metric line not containing $p$ and $p q=p r=p s$.

Received by the editors July 19, 1972 and, in revised form, October 31, 1972.

AMS (MOS) subject classifications (1970). Primary 52A50, 46B99.

Key words and phrases. Banach space, Chebyshev sets, metric lines, monotone property, rotundity, Young property.

(c) American Mathematical Society 1973 
Proof. The necessity of the condition is clear. To show its sufficiency suppose $M$ does not have the monotone property. In particular, assume for point $p$ and line $L$, the function $p x$ is not strictly monotone increasing as $x$ recedes from the foot $f$ of $p$ on $L$ along either half-line of $L$ determined by $f$. Then there are points $r, s$ of $L$ with $r$ between $f$ and $s$ and such that $p s \leqq p r$. Using the triangle inequality and continuity of the metric, there is a point $q$ of $L$ on the oppposite side of $f$ from $s$ such that $p q=p s$. If $p s=p r$ then $p, q, r, s$ is the desired quadruple. Otherwise, by continuity of the metric there is a point $r^{*}$ between $f$ and $r$ such that $p r^{*}=p s$, and the quadruple $p, q, r^{*}, s$ is of the desired type.

The proof of the main theorem makes strong use of the property that if $p, q, r$ are points of a normed linear space and $q^{\prime}, r^{\prime}$ are the algebraic midpoints of $p$ and $q, p$ and $r$ respectively, then $q^{\prime} r^{\prime}=\frac{1}{2} q r$. This property is referred to as the Young property due to the use of a similar property by W. H. Young [5] to distinguish between euclidean and hyperbolic geometry. The Young property, applied to metric midpoints, was more recently used in [1] to characterize Banach spaces among metric spaces with unique lines.

3. The characterization theorem. The main result is the following.

THEOREM 3.1. A Banach space has unique metric lines if and only if it has the monotone property.

Proof. Let $B$ be a Banach space with unique metric lines. If it does not have the monotone property, by Lemma 2.1 points $p, q, r, s$ exist with $q, r, s$ on a line $L$ not containing $p$, such that $p q=p r=p s$ and $q r+r s=q s$. Since $B$ has unique lines, each metric line is an algebraic line, and each metric midpoint is an algebraic midpoint of the same pair of points. Thus letting $m, n, m^{\prime}$ be the respective midpoints of $r$ and $s, r$ and $p$, and $r$ and $q$, it follows from the Young property that $m n=\frac{1}{2} p s$ and $n m^{\prime}=\frac{1}{2} p q$. By uniqueness of lines, $p, n, m$ are not collinear so $p m<p n+n m=p s$. Similarly $p m^{\prime}<p q=p s$. Letting $d$ be the larger of the real numbers $p m, p m^{\prime}$, we can find first points $u, v$ encountered on $L$ in proceeding from $r$ to $s, r$ to $q$ respectively, such that $p u=p v=d$. Clearly $u \neq v$, and for each point $x$ between $u$ and $v, p x>d$. Now if $m^{*}, n^{*}$ are the respective midpoints of $u$ and $v, p$ and $v$, application of the Young property as before yields

$$
p m^{*}<p n^{*}+n^{*} m^{*}=p u=d,
$$

a contradiction. Thus $B$ has the monotone property.

To prove the converse, suppose the Banach space $B$ does not have unique lines. Then there exists a quadruple of points $p, q, r, s$ such that $p q=q r=q s$ 
and $q$ is a common midpoint of $p$ and $r$ and of $p$ and $s$. Without loss of generality we may assume $q$ lies on the algebraic line of $p$ and $s$. Then the algebraic line of $p$ and $r$ does not contain $q$. Let $m$ be the algebraic midpoint of $r$ and $s$. Application of the Young property yields $q m=\frac{1}{2} p r=$ $q r=q s$, and by Lemma $2.1, B$ does not have the monotone property.

As an immediate consequence of the preceding it is now possible to list the following equivalents of the monotone property. (For definitions and further references see [3].)

THEOREM 3.2. Let $B$ be a Banach space (over the reals). The following are equivalent:

(i) The space $B$ has the monotone property.

(ii) The space $B$ has unique metric lines.

(iii) For each three points, $p, q, r$ of $B$ and each real number $a$, the equation $|p-[\lambda q+(1-\lambda) r]|=a$ has at most tiio real roots.

(iv) The unit spheres of $B$ are strictly convex.

(v) The space $B$ is rotund.

(vi) The one-dimensional subspaces of $B$ are Chebyshev sets.

(vii) The metric lines of $B$ are Chebyshev sets.

Proof. The equivalence of (i) and (ii) is Theorem 3.1. That of (i) and (iii), and hence that of (i) and (iv), is a result of Lemma 2.1. Equivalence of (iv), (v), and (vi) is known (see [3]). Since (vii) implies (vi) it suffices to observe that (vi) implies the monotone property from which it follows that the foot of any point on any metric line is unique, i.e., that the metric lines of $B$ are Chebyshev sets.

It should be noted that the techniques used in the proof of Theorem 3.1 yield an immediate proof of the well-known equivalence of (ii) and (iv) through simple applications of the Young property. Furthermore, as a consequence of the equivalence of (vii) and (ii), in order to prove that metric lines in a Banach space are not unique, it suffices to exhibit a single metric line and a single point whose foot on that line is not unique, a formal weakening of the requirement that some one-dimensional subspace not be a Chebyshev set.

\section{REFERENCES}

1. E. Z. Andalafte and L. M. Blumenthal, Metric characterizations of Banach and Euclidean spaces, Fund. Math. 55 (1964), 23-55. MR 29 \#2625.

2. L. M. Blumenthal, Theory and applications of distance geometry, Clarendon Press, Oxford, 1953. MR 14, 1009.

3. F. Cudia, Rotundity, Proc. Sympos. Pure Math., vol. 7, Amer. Math. Soc., Providence, R.I., 1963, pp. 73-97. MR 27 \#5106. 
4. R. W. Freese, Criteria for inner product spaces, Proc. Amer. Math. Soc. 19 (1968), 953-958. MR 37 \#3452.

5. W. H. Young, On the analytic basis of noneuclidean geometry, Amer. J. Math. 33 (1911), 249-286.

Department of Mathematics, University of Missouri, St. Louis, Missouri 63121

Department of Mathematics, Utah State University, Logan, Utah 84321 